ISSN 0103-5150

Fisioter. Mov., Curitiba, v. 24, n. 3, p. 437-443, jul./set. 2011 Licenciado sob uma Licença Creative Commons

\title{
Envelhecimento, depressão e quedas: um estudo com os participantes do Projeto Prev-Quedas
}

\author{
Aging, depression and falls: a study with the \\ participants of a falls prevention project
}

\author{
Hugo Leonardo Prata ${ }^{[a]}$, Edmundo de Drummond Alves Junior ${ }^{[b]}$, Fátima Lima Paula ${ }^{[c]}$, \\ Sabrina Manhães Ferreira ${ }^{[\mathrm{d}]}$
}

[a] Professor de Educação Física, Especialização, Grupo de Pesquisa Envelhecimento e Atividade Física - Universidade Federal Fluminense (UFF), Niterói, RJ - Brasil, e-mail: hugo.prata@uol.com.br

[b] Professor de Educação Física, Doutorado, professor da Universidade Federal Fluminense - Grupo de Pesquisa Envelhecimento e Atividade Física, Niterói, RJ - Brasil, e-mail: edmundodrummond@uol.com.br

[c] Fisioterapeuta, Mestrado, Grupo de Pesquisa em Envelhecimento e Atividade Física - Universidade Federal Fluminense (UFF), Niterói, RJ - Brasil, e-mail: fatima.lima.paula@gmail.com

[d] Professora de Educação Física, Graduação, Grupo de Pesquisa Envelhecimento e Atividade Física - Universidade Federal Fluminense (UFF), Niterói, RJ - Brasil, e-mail: sabrinamanhaes@bol.com.br

\section{Resumo}

Introdução: No Brasil a depressão é um problema de saúde pública, tendo 10 milhões de pessoas com esse diagnóstico. A queda de idosos é outro comprometedor da saúde, que muitas vezes apresenta uma combinação com a depressão. Objetivos: Verificar a associação entre estados depressivos e número de quedas. Materiais e métodos: Estudo seccional em que foi aplicado o GDS-15 e um questionário específico. Os dados foram trabalhados no programa R pelo teste Qui-Quadrado, ao nível de significância de 95\%. Foram incluídas idosas que participam do Projeto Prev-Quedas e o critério de exclusão foi apresentar deficiência auditiva não corrigida ou apresentar estágios avançados de distúrbios cognitivos e/ou doenças mentais que impedissem o entendimento das perguntas. Discussão e resultados: Foram entrevistadas 78 idosas, com idade média de 72 anos. 0 percentual de pessoas com possível quadro de depressão foi de 21,8\%. Não foi encontrada associação entre depressão e as variáveis idade, estado civil, morar com alguém $(p>0,05)$. Foi encontrada associação entre quedas e depressão $(\mathrm{p}<0,01)$. Conclusão: A tendência a estados depressivos encontrada na amostra foi considerada alta, acima da média nacional observada em 1999. A associação 
encontrada entre o quadro de depressão e um maior número de quedas sofridas pelos participantes pode indicar a necessidade de maior atenção das políticas públicas ao problema. A ausência de medidas preventivas estaria ligada ao pior padrão de qualidade de vida relacionada à saúde.

Palavras-chave: Envelhecimento. Depressão. Acidentes por quedas.

\begin{abstract}
Introduction: In Brazil depression is a public health problem. About 10 million people received this diagnosis in this country. The elderly falls are another serious health problem which is frequently related to depression. Objective: To verify association between depressive states and number of falls. Materials and methods: Sectional study which used the GDS-15 and a questionnaire related to falls. For the descriptive analysis, test of normality and forces of association (Chi-Squared) it was used the R program, version 2.8.1, with the confidence level of 95\%. Discussion and results: 78 old women were interviewed (mean age of 72 years). The percentage of depression was $21.8 \%$. Falls were significantly associated with depression $(p<0,01)$. Conclusions: The percentage of depression observed in the study was relatively high if we compare with data in Brazil in 1999. The association between depression and a greater number of falls can indicate the need of attention of public policy. The lack of prevention can be related to a worse quality of life.
\end{abstract}

Keywords: Aging. Depression. Accidental falls.

\section{Introdução}

0 envelhecimento populacional teve, durante $o$ século XX, o delineamento do que hoje se apresenta, o que tem como marca grandes preocupações no tocante à manutenção da saúde, aos gastos públicos com a aposentadoria e à dicotomia autonomia versus dependência $(1,2)$. Ao ser considerado como um problema social do momento, fica-se diante de uma complexidade de fatores nunca antes experimentada na história da humanidade, que desafia os elaboradores das políticas públicas (3). Daí a pertinência de se estabelecer estudos no campo do envelhecimento e, em especial, nos grandes agravos que contribuem para a diminuição da qualidade de vida dos que envelhecem, pois a cada ano aumentam as proporções dos que são considerados velhos. As projeções das pirâmides etárias (4) para as próximas décadas apontam para um processo de envelhecimento da população brasileira.

Os países desenvolvidos foram os primeiros a perceber os efeitos da transição demográfica, o que acabou por impor novas formas de encarar a velhice, surgindo um novo estilo de vida, que, entre outros pontos, sugere a importância de ser mais ativo. Nos próximos 40 anos, o Brasil passará a ter mais pessoas acima de 60 anos do que jovens com menos de 20 anos; em 2050 teremos em torno de 32 milhões de pessoas na faixa etária que hoje a Lei 8.842 (5) categoriza como "idoso". Isso é fruto das crescentes diminuições das taxas de fertilidade, que conjuntamente com a diminuição da taxa de mortalidade infantil e o consequente crescimento da expectativa de vida fez concentrar cada vez mais um contingente de pessoas idosas. As demandas geradas por essa nova realidade inquietam e devem ser prioritárias, uma vez que não basta apenas viver mais tempo, o desafio é no sentido de dar, a cada ano conquistado, melhores condições de enfrentar o envelhecimento e a velhice de forma autônoma e independente.

Com o crescimento do quantitativo de idosos aumentarão outras demandas que até pouco tempo eram desprezadas. Teremos no País um crescimento na prevalência de doenças crônico-degenerativas que mais afetam essa faixa etária, como, por exemplo, a depressão e as quedas, que ocorrem independentemente da idade, mas ao atingir os mais idosos as consequências passam a ser significativas $(6,7)$. Ambos são agravos multifatoriais, passíveis de medidas preventivas que visem a influir nos episódios que, com decorrer do envelhecimento, são mais recorrentes. Tanto a depressão como as quedas têm merecido pesquisas que procuram compreender melhor a relação desses problemas com o envelhecimento.

0 interesse desta investigação surge a partir das inquietações levantadas pelos profissionais que atuam 
em um projeto de intervenção que tem como objetivo central a prevenção de quedas (1). Esse projeto existe desde o ano de 2001, em uma perspectiva intergeracional e multiprofissional e objetiva, a partir da temática das quedas, de integrar à promoção da saúde dos que envelhecem estratégias tanto de proteção, prevenção e de educação para a saúde.

Apesar da probabilidade de certas doenças aumentarem com a idade, reforçamos a necessidade de não observar a velhice como um acúmulo de doenças, além de compreender que a aposentadoria não é a antecâmara da morte. Mesmo que o envelhecimento biológico indique diminuições e comprometimentos dos mais diversos, sabemos que muitos deles recebem forte contribuição do mau uso ou do desuso de determinadas qualidades físicas. Para manter a autonomia e a independência, e nesse caso a capacidade de ir e vir, é necessário que se trabalhe com foco em uma deambulação mais segura. Dessa forma, estamos de acordo com aqueles que enfatizam a necessidade da manutenção no melhor estado e os possíveis ganhos que ainda possam ocorrer. Sendo assim, ao tematizar as quedas, buscamos diminuir os fatores que comprometem a saúde dos que envelhecem.

Zimerman (8) sugere que o velho é um "mais", tem mais experiência, mais vivência, mais anos de vida, mais doenças crônicas, mais perdas, sofre mais preconceitos e tem mais tempo disponível. No momento em que utiliza mais sua experiência, a vivência adquirida ao longo de sua vida, aprende a viver com suas doenças crônicas, próprias da sua idade, e elabora suas perdas. Não esquecendo seus ganhos, dribla os preconceitos e aprende a utilizar o seu tempo.

A depressão é considerada um dos maiores problemas de saúde pública, atingindo cerca de 121 milhões de pessoas em todo o mundo. De acordo com a Organização Mundial da Saúde, a depressão representou a quarta maior causa de doenças mundiais em 2000, com projeção de se tornar a segunda em 2020 (9). No Brasil, segundo a Pesquisa Nacional por Amostra de Domicílios (PNAD) de 2008, 9,2\% das pessoas com 60 anos ou mais declararam sofrer de depressão (10).

Para Nuber (11), diferentemente do que a maior parte das pessoas pensa, a depressão não é uma doença da modernidade, sempre houve pessoas deprimidas. Nas narrativas históricas, há indicações de que há três mil anos os sacerdotes egípcios tratavam de uma doença que eles ainda não haviam conseguido definir, mas cuja descrição se aplicava exatamente ao caso da depressão: os sacerdotes observaram que depois de uma perda as pessoas sofriam de certo abatimento, bastante duradouro, que podia voltar em determinadas fases da vida. Hipócrates foi o primeiro a atribuir doenças psíquicas ao cérebro e o primeiro a descrever o fenômeno da "mania" e da "melancolia", descrição que ainda tem validade em nossos dias.

$\mathrm{Na}$ atualidade, pesquisas apontam para achados consistentes na literatura relacionados à depressão, entre eles: seu curso recorrente, o impacto do estresse e do fator de risco genético, o risco de suicídio, a multiplicidade de fatores e a prevalência em mulheres. Essa doença ocupa importante destaque não só pela sua gravidade, mas também por ocorrer em comorbidade com outras doenças (12).

Idosos com diabetes, hipertensão, doenças coronarianas e obesidade têm maior probabilidade de apresentar quadros de depressão. Sentimentos de frustração perante os anseios de vida não realizados e a própria história do sujeito, muitas vezes marcada por perdas progressivas, como a perda do(a) companheiro(a), dos laços afetivos e da capacidade de trabalho, sem contar a aposentadoria, que mina os recursos mínimos de sobrevivência, são fatores que comprometem a qualidade de vida e predispõem o idoso ao desenvolvimento da depressão (13).

Em outra classificação, não com menos importância, estão as quedas, que, assim como a depressão, são um acontecimento da mais alta importância na vida do idoso. Uma queda pode representar incapacidade, perda da função, levar à síndrome de imobilidade, morte, além de trazer prejuízo físico e psicológico, com a diminuição da autonomia e independência, vindo a aumentar o custo social (14).

A queda é um evento que acontece com certa frequência, é limitante, podendo ser um indício de algo errado com a saúde do idoso, um indicativo da eminência de uma doença ainda não diagnosticada. Em última análise, uma queda pode ser considerada como um marcador de fragilidade, morte, institucionalização e de declínio da saúde dos mais idosos $(15,16)$.

Os episódios das quedas envolvendo adultos idosos ultrapassam as questões sanitárias e sociais, eles repercutem no ambiente familiar, no aspecto econômico, afetam o indivíduo tanto pelo aspecto físico como psicologicamente, principalmente em função da sua recorrência, como é mostrado em uma pesquisa longitudinal prospectiva realizada com 336 idosos norte-americanos não institucionalizados que tinham 
a idade de 75 anos ou mais (17). Nesse estudo verificou-se que, na população investigada por um ano, $32 \%$ relataram que caíram pelo menos uma vez nos dois últimos anos. Dos indivíduos que relataram queda durante o estudo, 46\% caíram uma vez, 29\% caíram duas vezes, $25 \%$ caíram pelo menos três vezes.

No Brasil, Siqueira et al. (18) realizaram um estudo seccional com uma amostra de 4003 indivíduos com 65 anos ou mais. Investigou-se 41 municípios com mais de cem mil habitantes localizados em sete estados, encontrou-se uma prevalência de quedas de ordem de $34,8 \%$.

A maior suscetibilidade dos idosos sofrerem quedas se deve, entre outras, à alta prevalência de comorbidade presente nessa população. Associa-se também às alterações decorrentes do processo de envelhecimento, às condições patológicas agudas e crônicas e ao uso de medicamentos. Bloch et al. (19) verificaram associação entre quedas e utilização de psicotrópicos em idosos. Kerse et al. (20) realizaram um estudo em que identificaram associação significativa entre uso de antidepressivos e quedas de idosos.

Um estudo realizado na Nova Zelândia encontrou relação entre as pessoas adultas que sofrem de depressão e tomam medicamentos para o tratamento da doença com o aumento das chances de virem a sofrer uma queda (21).

0 objetivo de nossa pesquisa foi responder a questões que rondam a relação entre esses dois agravos: quedas e quadros depressivos, e para esse fim foi utilizado como base um grupo de idosos que frequenta um projeto de prevenção de quedas na Universidade Federal Fluminense (1).

\section{Materiais e métodos}

É recorrente a utilização de questionários estandardizados para ajudar no diagnóstico e medir o grau de depressão. 0 questionário utilizado nesta pesquisa foi a escala de depressão geriátrica (GDS) 15 adaptada $(22,23)$ que contém 15 questões fechadas, com alternativas positivas e negativas, relacionadas ao aspecto emocional e cognitivo. Os pontos de corte são: normal ou sem depressão (N) - inferior a 5 pontos; depressão leve (L) - entre 5 e 10 pontos; depressão grave (G) - acima de 10 pontos.

A população foi composta por 84 indivíduos pertencentes ao Projeto Prev-Quedas. Os critérios utilizados para inclusão foram: ser mulher, ser participante do projeto, concordar em assinar o Termo de Consentimento Livre e Esclarecido. Já para exclusão foram: declarar pessoalmente, ou por meio de informante, que era portador de deficiência auditiva não corrigida ou apresentar estágios avançados de distúrbios cognitivos e/ou doenças mentais que impedissem o entendimento das perguntas. As idosas que usavam aparelho auditivo bem adaptado participaram do estudo.

A coleta dos dados foi realizada no mesmo local onde ocorriam as atividades do projeto, as entrevistas foram realizadas individualmente. Os dados foram analisados no programa R, com nível de significância de $95 \%$. Foi testada a normalidade dos dados pelo teste de 'Shapiro'. A força de associação foi verificada pelo teste do Qui-Quadrado. A pesquisa foi realizada no ano de 2008, com aprovação pelo Comitê de Ética da Faculdade de Medicina da Universidade Federal Fluminense, sob o número 0154.258.00007.

\section{Resultados}

Dos 84 participantes do Projeto Prev-Quedas, quatro foram excluídos por problemas de distúrbios cognitivos ou de déficit auditivo e dois por serem do sexo masculino. Sendo assim, não houve perdas e o número de participantes da pesquisa foi 78. A idade variou de 53 a 89 anos, com média em 72,0 e desvio-padrão $(7,5)$. Quanto ao estado civil, a maior prevalência foi a de viuvez.

Em relação ao estado depressivo, 17,9\% dos indivíduos que responderam ao questionário declararam que tinham depressão. 0 resultado do GDS foi que $21,8 \%$ têm o quadro de possível depressão leve e 78,2\%, de depressão normal ou sem depressão. A Tabela 1 mostra a frequência de depressão declarada e do resultado do GDS ( $p<0,001)$.

Em relação ao número de quedas (Tabela 2), 32\% dos participantes declararam terem caído no último ano, e 53 , que correspondem a $68 \%$, ou não caíram ou não se lembram de terem caído. Conforme se percebe na mesma tabela, entre os que caíram no último ano, $10,3 \%$ tiveram só uma queda, $7,7 \%$ sofreram duas quedas e $14,1 \%$ tiveram três quedas ou mais.

\section{Discussão}

A prevalência de quedas no último ano, encontrada na presente pesquisa, foi de $32,0 \%$ em amostra 
de participantes do Projeto Prev-Quedas. O resultado da pesquisa mostrou similaridade com outras pesquisas encontradas na literatura. Estudos realizados nos EUA e na Turquia relatam prevalência de quedas em idosos de 30\% (24) e 31,9\% (25), respectivamente. É possível confrontar, ainda, os resultados da presente pesquisa com outro estudo realizado no Brasil, em que 34,8\% dos idosos investigados sofreram queda no ano anterior à entrevista (18). É presumível que quando comparado com pesquisas do mesmo país existam maiores chances de aproximação das particularidades, mesmo sabendo que o Brasil é um país continental e bem distinto entre as regiões, principalmente no que tange às questões sociais.

A prevalência da depressão na amostra do estudo foi de $21,8 \%$. Segundo Oliveira et al. (2006), a prevalência de depressão em idosos frequentadores de centros de convivência em uma cidade do Distrito Federal foi de 31\% (26). Esses autores utilizaram a mesma escala utilizada neste estudo. Blay e Bickel (27), em pesquisa realizada no Brasil, indicam, como alta prevalência de depressão, $14,3 \%$ na população de 65 anos ou mais que vive na comunidade. Uma

Tabela 1 - Prevalência de quadro depressivo diagnosticado e resultado do GDS entre os participantes do Projeto Prev-Quedas n. 78

\begin{tabular}{lcc}
\hline & N & $\%$ \\
\hline Depressão declarada & 14 & $17,9 \%$ \\
Depressão não declarada & 64 & $82,1 \%$ \\
Resultado GDS & 17 & $21,8 \%$ \\
\hline
\end{tabular}

Fonte: Dados da pesquisa.

Tabela 2 - Frequência de quedas declarada pelos participantes do Projeto Prev-Quedas n. 78

\begin{tabular}{lcc}
\hline & N & $\%$ \\
\hline Uma queda no último ano & 08 & 10,3 \\
Duas quedas no último ano & 06 & 7,7 \\
Três quedas ou mais no último ano & 11 & 14,1 \\
Não caiu no último ano & 53 & 68 \\
\hline
\end{tabular}

Fonte: Dados da pesquisa. hipótese para essas diferenças dos valores pode ser o aumento da prevalência ao longo do tempo por fatores que não foram objeto do presente estudo. Outra hipótese levantada é a de que existam diferenças entre a prevalência de depressão entre as cidades em razão de divergências socioeconômicas.

Um número significativo de estudos internacionais estima que a prevalência de sintomas depressivos na população acima de 65 anos de idade vivendo na comunidade varia entre 10,3\% e 13,5\% (28). Ao contrário do que sugerem pesquisas nacionais e internacionais que relatam a maior incidência de depressão entre os idosos que vivem sozinhos, não encontramos essa relação de depressão versus morar sozinho $(p>0,05)$, nem entre as variáveis idade e estado civil.

Não foi analisada na pesquisa a relação de ingestão de medicamentos antidepressivos com o aumento do número de quedas. No entanto, isso não inviabiliza essa relação, pois o estudo se baseou na literatura de trabalhos que defendem a ideia de que a depressão acrescida de medicamentos para tratar os seus sintomas pode predispor quedas $(29,30)$. Com base na pesquisa realizada por esses autores, pode-se levantar a hipótese de que a associação encontrada na presente pesquisa, entre prevalência de quedas e depressão, pode se dar em função da ingestão de medicamentos ou, ainda, por diversas outras razões, apontadas na literatura, que os distúrbios depressivos podem ocasionar, como: indiferença ao meio ambiente, adulteração do nível da atenção, redução do comprimento da passada, diminuição da energia, diminuição da autoconfiança, reclusão, inatividade e perdas cognitivas.

Tanto a depressão quanto as quedas, apesar de serem situações totalmente diferentes, guardando cada uma as suas particularidades, podem ser caracterizadas como um problema de saúde pública, pois existem em alta prevalência na sociedade contemporânea, sendo possível observar um impacto tanto no indivíduo quanto na sociedade, gerando elevado custo financeiro para os sistemas de saúde (31). Sugere-se um número maior de investigações sobre os temas depressão e quedas, já que existe uma lacuna de publicações sobre o assunto. Além disso, sugere-se também maior atenção das autoridades competentes, já que essas alterações estão ligadas diretamente ao pior padrão de qualidade de vida relativa à saúde. 


\section{Referências}

1. Alves ED Jr. Envelhecimento e vida saudável. Rio de Janeiro: Apicuri; 2010.

2. Veras RP, Caldas CP. Produção de cuidados à pessoa idosa. Ciência \& Saúde Coletiva. 2008;13(4):1104.

3. Lenoir R. Objeto sociológico e problema social. In: Champagne P, Lenoir R, Merllé D, Pinto L. Iniciação à prática sociológica. Petrópolis: Vozes; 1998. p. 59-106.

4. Population Reference Bureau. [acesso em 10 maio 2009]. Disponível em: http://www.prb.org/Publica tions/populationBulletins/2008/aging.aspxwww. prb.org/Publications/PopulationBulletins/2009/ aging.aspx

5. Brasil, Senado Federal Brasileiro. Lei Ordinária 1994. Brasília; 1994. [acesso em 15 nov. 2008]. Disponível em: http://www.senado.gov.br

6. Fontaine R. Psicologia do envelhecimento. Lisboa: Climepsi; 2000.

7. Perracini MR, Ramos LR. Fatores associados a quedas em uma coorte de idosos residentes na comunidade. Rev Saúde Pública. 2002:36(6):93-9.

8. Zimerman L. Velhice - aspectos biopsicossociais. Porto Alegre: Artes Médicas; 2000.

9. World Health Organization. Ficha descritiva: depressão. 2008. [acesso em 20 nov. 2008]. Disponível em: http://www.who.int/mental_health/management/ depression/definition/en/

10. Brasil, Instituto Brasileiro de Geografia e Estatística, Pesquisa Nacional por Amostra de Domicílios, 2008. Síntese de Indicadores Sociais - Uma análise das condições de vida da população brasileira, 2010. [acesso em 20 nov. 2008]. Disponível em: http:// www.ibge.gov.br

11. Nuber U. Depressão: a doença mal compreendida. São Paulo: Pensamento; 1998.

12. Miguel F, Almeida OP. Aspectos psiquiátricos do envelhecimento. In: Carvalho F, Netto MP, Geriatria: fundamentos, clínica e terapêutica. São Paulo: Atheneu; 2000. p. 63-80.

13. Siegler I, Poon W, Madden D, Welsh K. Aspectos psicológicos do envelhecimento normal. In: Busse E, Blazer D. Psiquiatria geriátrica. Porto Alegre: Artmed; 1999. p. 119-39.
14. Centers for Disease Control and Prevention (CDC). Falls among older adults: an overview, 2010. [acesso em 5 fev. 2011]. Disponível em: http://www.cdc. gov/HomeandRecreationalSafety/Falls/adultfalls. html

15. Tinetti M. Prevention of falls and fall injuries in elderly persons: a research agenda. Prev Med. 1994;23(5): 756-62.

16. Fabrício SCC, Rodrigues RAP, Costa ML Jr. Causas e conseqüências de quedas de idosos atendidos em hospital público. Rev Saúde Pública. 2004;38(1):93-9.

17. Tinetti ME, Doucette J, Claus E, Marottoli R. Risk factors for serious injury during falls by older persons in the community. J Am Geriatr Soc. 1995;43(11): 1214-21.

18. Siqueira FV, Facchini LA, Piccini RX, Tomasi E, Silveira DS, Vieira V, et al. Prevalência de quedas em idosos e fatores associados. Rev Saúde Pública. 2007; 41(5):749-56.

19. Bloch $F$, Thibaud $M$, Dugué $B$, Brèque $C$, Rigaud $A S$, Kemoun G. Psychotropic drugs and falls in the elderly people: updated literature review and metaanalysis. J Aging Health. 2011;23(2):329-46.

20. Kerse N, Flicker L, Pfaff JJ, Draper B, Lautenschlager NT, Sim M, et al. Falls, depression and antidepressants in later life: a large primary care appraisal. 2008 PLoS ONE. 2008;3(6):e2423.

21. Hartikainen S, Lönnroos E, Louhivuori K. Medication as a risk for falls: critical systematic review. J Gerontol A Biol Sci Med Sci. 2007;62(10):1172-81.

22. Paradela E, Lourenço R, Veras R. Validação da escala de depressão geriátrica em um ambulatório geral. Rev Saúde Pública. 2005;39(6):918-23.

23. Yesavage JA, Brink TL, Rose TL, Lum O, Huang V, Adey M, et al. Development and validation of a geriatric screening scale. J Psychiatr Res. 1982-1983; 17(1):37-49.

24. Tinetti ME, Baker DI, Mcaay G, Clauss EB, Garrett P, Gottschalk M, et al. A multifactorial intervention to reduce the risk of falling among elderly people living in the community. N Engl J Med. 1994;331(13):821-7.

25. Evci E, Ergin F, Beser E. Home accidents. In the elderly in Turkey. Tohoku J Exp Med. 2006;209(4):291-301. 
26. Oliveira DAAP, Gomes L, Oliveira RF. Prevalência de depressão em idosos que freqüentam centros de convivência. Rev Saúde Pública. 2006;40(4):734-6.

27. Blay SL, Bickel H, Cooper B. Mental illness in cross: national perspective: results from a Brazilian and German community survey among the elderly. Soc Psychiatry Psychiatr Epidemiol. 1991;26(6):245-51.

28. Snowdon J. How high is the prevalence of depression in old age. Rev Bras Psiquiatria. 2002;24(suppl.):42-7.

29. Turcu A, Toubin S, Mourey F, D’Athis P, Manckoundia P, Pfitzenmeyer P. Falls and depression in older people. Gerontology. 2004;50(5):303-8.
30. Hartikainen S, Lönnroos E, Louhivuori K. Medication as a risk for falls: critical systematic review. J Gerontol A Biol Sci Med Sci. 2007;62(10):1172-81.

31. Costa JSD, Victora CG. 0 que é um problema de saúde pública. Rev Bras Epidemiol. 2006;9(1):144-6.

Recebido: 08/01/2011

Received: 01/08/2011

Aprovado: 18/04/2011

Approved: 04/18/2011 